\title{
STELLAR ENCOUNTERS IN DENSE SYSTEMS
}

\author{
MELVYN B. DAVIES \\ Institute of Astronomy \\ Cambridge, UK
}

\section{Introduction}

The number density of stars in the solar neighbourhood is sufficiently low that encounters between two stars will be extremely rare. However, in the cores of globular clusters, and glactic nuclei, number densities are sufficiently high $\left(\sim 10^{5} \mathrm{stars} / p c^{3}\right.$ in some systems $)$ that encounter timescales can be comparable, or even less than, the age of the universe. In other words, a large fraction of the stars in these systems will have suffered from at least one close encounter or collision in their lifetime.

The cross section for two stars, having a relative velocity at infinity of $v_{\infty}$, to pass within a distance $R_{\min }$ is given by

$$
\sigma=\pi R_{\min }^{2}\left(1+\frac{v^{2}}{v_{\infty}^{2}}\right)
$$

where $v$ is the relative velocity of the two stars at closest approach in a parabolic encounter (i.e. $\left.v^{2}=2 G\left(m_{1}+m_{2}\right) / R_{\min }\right)$. The second term is due to the attractive gravitational force, and is referred to as gravitational focussing. In the regime where $v \ll v_{\infty}$ (as might be the case in galactic nuclei with extremely high velocity dispersions), we recover the result, $\sigma \propto$ $R_{\min }^{2}$. However, if $v \gg v_{\infty}$ as will be the case in systems with low velocity dispersions, such as globular clusters, $\sigma \propto R_{\min }$.

One may estimate the timescale for a given star to undergo an encounter, $\tau_{\text {coll }}=1 / n \sigma v$. For the two extreme cases mentioned above, we thus obtain

$$
\tau_{\text {coll }}=7 \times 10^{10} \mathrm{yr}\left(\frac{10^{5} p c^{-3}}{n}\right)\left(\frac{v_{\infty}}{10 k m / s}\right)\left(\frac{R_{\odot}}{R_{\min }}\right)\left(\frac{M_{\odot}}{M}\right) \text { for } v \gg v_{\infty}
$$




$$
\tau_{\text {coll }}=7 \times 10^{12} \mathrm{yr}\left(\frac{10^{5} p c^{-3}}{n}\right)\left(\frac{100 k m / s}{v_{\infty}}\right)\left(\frac{R_{\odot}}{R_{\min }}\right)^{2} \text { for } v \ll v_{\infty}
$$

where $n$ is the number density of single stars of mass $m$. Thus for globular clusters, $\tau_{\text {coll }} \propto 1 / R_{\min }$. For an encounter between two single stars to be hydrodynamically interesting, we typically require $R_{\min } \leq 3 R_{\odot}$ (see for example, Davies, Benz \& Hills 1992). For an encounter between a binary and a third, single star, we require that $R_{\min } \simeq d$, where $d$ is the semi-major axis of the binary. Even for a binary with $d \sim 1 A U\left(\equiv 216 R_{\odot}\right)$, we see that $\tau_{\text {coll }} \ll 10^{10}$ years in the core of a dense globular cluster. Thus encounters between binaries and single stars will be important in globular clusters even if the binary fraction is $\sim 5 \%$. In encounters between two binaries, we again require the two systems to pass within $\sim d$ of each other. Hence binary/binary encounters will dominate over binary/single encounters only if the binary fraction is $\geq 30 \%$. The issue of the binary fraction in the core of a globular cluster is thus an important one. Unfortunately, the number is not well known.

\section{Dynamical Evolution of Globular Clusters}

Neglecting for a moment the role of binaries, a globular cluster will evolve dynamically in the following way. Stars in the halo will be heated by stars from the cluster core by two-body scattering, causing some to escape from the cluster (i.e. evaporate). Losing energy, the core contracts, and its velocity dispersion increases. In thermodynamic parlance, the system has a negative heat capacity with the velocity dispersion being equivalent to a temperature. Thus as the core contracts, the temperature gradient, and in turn the rate of energy transfer to the stars in the halo, increases. It was shown that such a system would reach an infinite central density in a finite time (Cohn 1980). This is known as the gravothermal catastrophe (Lynden-Bell \& Wood 1968).

Binaries will be an important source of energy against the collapse of the core of a globular cluster. Hard binaries encountering a single, third star tend to be further hardened. In other words, the binary becomes more tightly bound, the shift in potential energy being manifested as an increase in the kinetic energies of the binary and single star, i.e. the stars receive kicks. This helps support a globular cluster in two ways. Firstly, the velocity dispersion of stars is increased by the kicks they receive. Further, if any stars are ejected, then the binding energy of the cluster is reduced as it contains fewer stars. 


\section{Stellar Exotica within Globular Clusters}

Low-mass X-ray binaries are observed in excessive numbers in globular clusters compared to the rest of our galaxy, given the fraction of stars in globular clusters. These systems may be formed via tidal capture of a neutron star by either a main-sequence star or red giant (Fabian, Pringle \& Rees 1975). Alternatively, they may be produced from primordial binaries by some evolutionary path that may include neutron stars being exchanged into a binary in some encounter. The relative frequency of these two paths will depend on the make-up of the primordial binary population and their evolution due to encounters with other binaries and single stars. It is also far from clear whether a system formed from tidal capture can survive as a binary or will form a single, merged object (Davies et al. 1992, and references contained within).

Millisecond pulsars (MSPs) have also been observed in large numbers in globular clusters (see, for example, Manchester et al. 1991). Under the standard model, MSPs are produced in Low-Mass X-ray Binaries (LMXBs) where the neutron star is spun-up as material is accreted from the Rochefilled companion. However observations seem to suggest that there are far more MSPs than LMXBs which, given their comparable expected lifetimes, poses a problem for the standard model (Kulkarni et al. 1990). This embarrassing profusion of MSPs coupled with the relative sparsity of LMXBs would seem to represent the nemesis of the standard model. Perhaps MSPs are formed without passing through a precursor LMXB phase, from systems containing neutron stars smothered by the remains of a main-sequence star after a collisions between two such stars, for example.

One might expect that white dwarfs experience encounters similar to neutron stars, some of which may lead to Cataclysmic Variables (CVs). As the number of LMXBs in globular clusters is large, one might expect to see copious numbers of CVs. Such objects are much harder to find, however, although CV candidates have been found in NGC 6397 by imaging the cluster in $\mathrm{H} \alpha$ (Cool et al. 1995), and confirmed through spectroscopy (Grindlay et al. 1995). CV candidates have also been seen in NGC 6752 in the X-ray (Cool 1993), and using $\mathrm{H} \alpha$ imaging (Bailyn et al. 1995).

Blue stragglers are positioned on the upper end of the main-sequence beyond the present day turn-off mass. They have been observed in many globular clusters, including: 47 Tuc (Paresce et al. 1991), NGC 6397 (Auriere et al. 1990) and M30 (Yanny et al. 1994, Guhathakurta et al. 1996). These stars may have formed from the merger of two lower-mass main-sequence stars either in an encounter between two single stars or in encounters involving binaries when two main-sequences collide and merge as part of the encounter. Such mergers may occur during an encounter between a binary 
and single star, as will be discussed in $\S 6$. The fraction of blue stragglers in binaries may thus be an important diagnostic for the binary fraction for globular clusters.

\section{Encounters between Two Single Stars in Globular Clusters}

We begin by considering encounters between two single stars, applicable to globular clusters where the parameter space is relatively small. The velocity dispersion is low, and essentially all stars were created in one epoch. Thus the maximum mass of primordial main-sequence stars today is well defined, as is the mass of any red giants involved in encounters today. Early 1D and 2D work was performed by Mathis (1967), De Young (1968) and Seidel and Cameron (1972). Three dimensional studies had to wait until the development of faster computers. A method known as Smooth Particle Hydrodynamics (SPH) has been applied to study stellar collisions (Benz and Hills 1987, 1992; Davies, Benz and Hills 1992, 1993, 1994; Hernquist and Goodman 1992; Rasio and Shapiro 1991, 1992). Additionally, encounters have been simulated using the PPM method (Ruffert and Müller 1991). The results of these studies can be summarised with three numbers: the capture radius, $R_{\text {capt }}$, which is the minimum distance required for a given pair of stars to form a bound system assuming a relative speed at infinity of $10 \mathrm{~km} / \mathrm{s}$; the merger radius, $R_{\text {merg }}$, which is the largerst value of the minimum distance of an encounter which produces a single merged object; and a typical value for the amount of material ejected from the system, $M_{\text {lost }}$.

Below we review the current understanding of encounters between various stellar species, considering encounters involving main-sequnece stars and red giants of turn-off mass $\left(0.8 \mathrm{M}_{\odot}\right)$. We discuss encounters between two main-sequence stars (MS/MS encounters), encounters between a mainsequence star and a more compact star, such as a white dwarf or neutron star (MS/CO encounters), and encounters between a red giant and smaller stars (RG/CO encounters).

\subsection{MS/MS ENCOUNTERS}

As alluded to in $\S 3$, collisions between two main-sequence stars remains a viable route to produce the observed blue stragglers. Simulations yield values of $R_{\text {capt }} \sim 3 R_{\mathrm{ms}}$, and provide a lower limit of $R_{\text {merg }} \sim 2 R_{\mathrm{ms}}$. The mass lost from the system on the initial impact is small, typically $M_{\text {lost }} \leq$ $0.01 M_{\mathrm{ms}}$. Early work suggested that the merged stars would be well mixed (Benz and Hills 1987). More recently simulations, using a more centrally concentrated model for the main-sequence stars, seem to suggest that the material in the cores will not (at least initially) mix with the envelope gas (Lombardi, Rasio and Shapiro 1995). The subsequent evolution of the 
merged objects remains an open question: will they expand significantly up the Hyashi track to come into thermal equilibrium? Will they become deeply convective and thus mix the material at some later time? Some clue to their subsequent evolution may be found in observations of blue stragglers in binaries: if we see such objects in hard, but eccentric, binaries then we may deduce that the stars didn't expand very much after formation. Conversely, if we see a blue straggler in a wide, but circular binary, this may suggest that the star had a much larger radius at an earlier time.

\subsection{MS/CO ENCOUNTERS}

Simulations yield values of $R_{\text {capt }} \sim 3.5 R_{\mathrm{ms}}$, and provide a lower limit of $R_{\text {merg }} \sim 1.8 R_{\mathrm{ms}}$. The mass lost from the system on the initial impact is larger than seen in MS/MS encounters, with $M_{\text {lost }} \sim 0.1 M_{\text {ms. }}$. In the case of encounters involving a neutron star, the subsequent evolution of merged systems is extremely unclear. It has been speculated that a small amount of material will be accreted by the neutron star, spinning it up, possibly to millisecond periods, whilst the enveloping gas prevents it from being visible as an X-ray object. Such a path way might help explain the apparent birthrate problem between LMXBs and MSPs, providing a sufficient number of smothered neutron star systems can be produced relative to clean binaries. In the case of encounters involving white dwarfs, any merged system seems likely to evolve into a red giant.

\subsection{RG/CO ENCOUNTERS}

Simulations yield values of $R_{\text {capt }} \sim 2.5 R_{\text {rg }}$, and provide a lower limit of $R_{\text {merg }} \sim 2 R_{\mathrm{rg}}$. The mass lost from the system on the initial impact $\sim 0.1 M_{\mathrm{rg}}$. Thus the vast majority of encounters would seem to produce merged systems, containing the red-giant core (essentially a white dwarf) and the impactor engulfed in a common envelope of gas. In such a system, the white dwarf and impactor will spiral together as the common gaseous envelope is ejected. The final separation of the two stars may be estimated by simply equating the binding energy of the envelope with the change in binding energy of the two stars, within some efficiency $\alpha_{\text {ce }}$ (typically taken to be 0.2-0.4 [Taam \& Bodenheimer 1989]), Such a common envelope phase will be of great use in producing hard binaries in globular clusters. For systems containing two compact objects, inspiral will occur due to angular momentum loss from gravitational radiation. A system will merge in $\sim 10^{10}$ years if the initial separation (after a common envelope phase) $\leq 3 R_{\odot}$ (Landau \& Lifshitz 1962), as will often be the case for post-CE binaries. 


\section{Encounters between Two Single Stars in Galactic Nuclei}

Less computational work has been done on encounters that will occur in galactic nuclei. The relevant parameter space is much larger than for globular clusters, with a wide range of relative velocities being important. As the relative velocity increases, encounters involving larger stars become progressively more important as the effect of gravitational focussing is reduced. In other words, encounters involving red giants may be more important than encounters involving main-sequence stars if the velocity dispersion $\geq$ $600 \mathrm{~km} / \mathrm{s}$, as will be the case in the very centres of some active galaxies.

Some work considering encounters between main-sequence stars has been performed (Benz and Hills, 1987, 1992; Lai et al. 1993; and Davies 1995 [in preparation]).

\section{Encounters between Binaries and Single Stars}

Encounters between binaries and single stars treating the stars as point masses have been considered in numerous works (Hut 1994, and references contained therein). Some allowance has also been made for the finite size of the stars in three-body simulations (see for example, Sigurdsson \& Phinney 1994). Cleary \& Monaghan (1990), and Davies, Benz \& Hills (1993, 1994) also considered the hydrodynamical effects by performing some encounters using a smoothed particle hydrodynamics (SPH) code.

Encounters between binaries and single stars lead to three main outcomes: the incoming star replaces one of the original components forming a new, detached, binary in a so-called clean exchange, the incoming star simply hardens the binary without any exchange occuring - a so-called flyby, or a merger between two of the stars occurs, where the product of the merger may or may not remain bound to the third star.

Before discussing the various cross sections, we will mention what has become known as Heggie's Law, namely that hard binaries get harder, and weak binaries are broken up (Hut 1983). In this context, hard means a binary that is sufficiently tightly bound that the orbital speeds of the two stars in the binary are much larger than the relative speed, at infinity, of the system and the third star. The dividing line between these two regimes is a function of the masses of the stars, and the relative velocity of the binary and the single stars it encounters. For example, with solar-mass stars, and a velocity dispersion of $10 \mathrm{~km} / \mathrm{s}$ (typical for a globular cluster), the water shed occurs at a semi-major axis of $\sim 10 \mathrm{AU}$. In a somewhat dense cluster such as 47 Tuc, we would expect surviving binaries to be hardened today from this separation to values $\sim 0.2-0.5 \mathrm{AU}$ (Hut, McMillan \& Romani 1992). 
The cross section for a single star to pass within a distance $R_{\min }$ of the center of mass of a binary is given by $\sigma=\pi R_{\min }^{2}\left(1+V_{c}^{2} / V_{\infty}^{2}\right)$, where $V_{\infty}$ is the relative speed at infinity, and $V_{c}$ is the relative speed at which the system has zero total energy and is given by $V_{c}^{2}=F\left(M_{1}, M_{2}, M_{3}\right) / d=$ $G M_{1} M_{2}\left(M_{1}+M_{2}+M_{3}\right) / M_{3}\left(M_{1}+M_{2}\right) d$, where $M_{1}$ is the mass of the primary, $M_{2}$ the mass of the secondary and $M_{3}$ is the mass of the incoming, single star. In order for an exchange encounter to occur where the impacting star replaces one of the binary components, one might expect $R_{\min } \sim d$, where $d$ is the separation of the two components in the original binary. For a binary of separation $\sim 10 \mathrm{AU}, V_{c} \simeq 10 \mathrm{~km} / \mathrm{s}$ (a typical value for the velocity dispersion in a globular cluster). Hence for hard binaries in globular clusters (where $d \sim 0.1-0.5 \mathrm{AU}$ ), $V_{c} \gg V_{\infty}$, and the exchange cross section can be written as

$$
\sigma_{\mathrm{ex}}=k_{\mathrm{ex}}\left(q_{1}, q_{2}\right) \pi d^{2} \cdot \frac{G M_{1} F\left(q_{1}, q_{2}\right)}{d} \cdot \frac{1}{V_{\infty}^{2}}
$$

where $q_{1}=M_{2} / M_{1}, q_{2}=M_{3} / M_{1}$, and $F\left(q_{1}, q_{2}\right)=q_{1}\left(1+q_{1}+q_{2}\right) / q_{2}\left(1+q_{1}\right)$. The constant $k_{\text {ex }}\left(q_{1}, q_{2}\right)$ has to be determined through numerical simulations. Once we have the value of $k_{\text {ex }}$ for a range of values of $q_{1}$ and $q_{2}$, we are able to compute the exchange cross section for any reasonable binary-single star encounter (Davies 1995).

Hut and Inagaki (1985) found that the cross section for any two stars to pass within some minimum distance can be written in the following form,

$$
\sigma\left(R<R_{\min }\right)=k_{\mathrm{rmin}}\left(q_{1}, q_{2},\right) \pi d^{2} \frac{G M_{1} F\left(q_{1}, q_{2}\right)}{d} \cdot \frac{1}{V_{\infty}^{2}}\left(\frac{R_{\min }}{d}\right)^{\gamma}
$$

where both $k_{\text {rmin }}$ and $\gamma$ can be found through simulations of encounters, with $\gamma \sim 0.5$ for encounters involving stars of equal masses. A form suggested for the differential exchange cross section is (Heggie 1975, Hut 1984)

$$
\frac{d \sigma}{d \Delta}=3.5 k_{\mathrm{ex}}\left(q_{1}, q_{2}\right) \pi d^{2} \cdot \frac{G M_{1} F\left(q_{1}, q_{2}\right)}{d} \cdot \frac{1}{V_{\infty}^{2}}(1+\Delta)^{-4.5}
$$

where $\Delta=\delta E_{\text {bin }} / E_{\text {bin }}$, the relative shift in the binding energy of the binary. The values predicted from the above equation are in close agreement with those obtained from three-body runs. On average an encounter will harden a binary by $20-40 \%$, the change in potential energry being seen in a boost to the binary's kinetic energy. We can thus compute the kick velocity given to a binary for each encounter. Very few binaries with separations $\sim 0.2 \mathrm{AU}$ will receive kicks sufficient to eject them from a typical globular cluster $\left(V_{\text {kick }} \sim 40 \mathrm{~km} / \mathrm{s}\right)$. Rather, it is more interesting to consider a kick velocity 


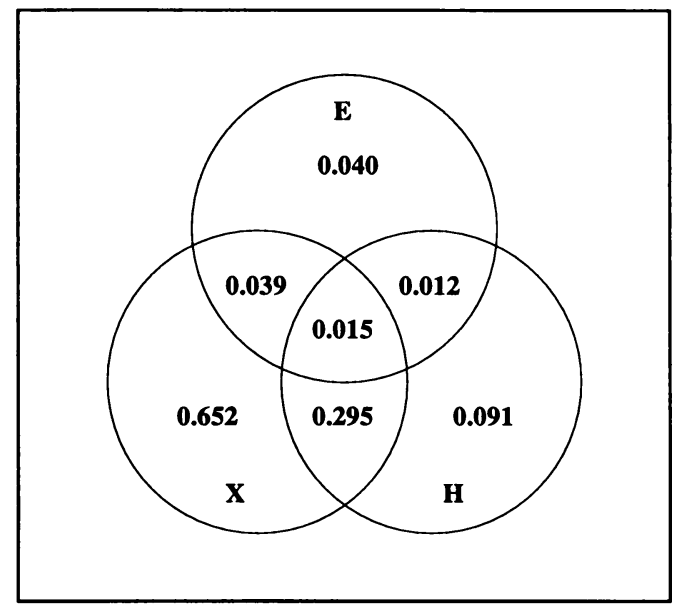

Figure 1. Relative cross sections for the various combinations of exchange (X), ejection $(\mathrm{E})$, and the necessity to include hydrodynamical effects $(\mathrm{H})$, calculated from a series of 7000 three-body simulations of encounters between a binary of separation $60 R_{\odot}$ and eccentricity $e=0.5$ containing an $0.8 M_{\odot}$ main-sequence star and a $1.4 M_{\odot}$ neutron star, and a $1.4 M_{\odot}$ neutron star. The cross sections are renormalized in such a way that the total cross section for processes leading to exchange is unity.

$\sim 20 \mathrm{~km} / \mathrm{s}$ which would be sufficient to propel the binary into an orbit that takes it far outside the core of a typical globular cluster.

We can compute the cross section for the following three processes: ejection from the globular cluster core, exchange, and strong hydrodynamical encounters (i.e. two stars pass within $3 R_{\mathrm{ms}}$ ). A convenient way to illustrate the fruit of these calculations is in a Venn diagram. In such a diagram, the three circles represent the three processes, and, for example, the number placed in the region common to all three circles is the cross section for exchange and ejection and hydrodynamical encounters, renormalized in such a way that the cross section for exchange is unity. Such a Venn diagram is illustrated in Figure 1 for encounters between an eccentric binary $(e=0.5$, with a semi-major axis, $d=0.2 \mathrm{AU}$ ) containing stars of mass $1.4 M_{\odot}$ and $0.8 M_{\odot}$, and third star of mass $1.4 M_{\odot}$. We see from this figure that some $30 \%$ of encounters which were labelled as exchanges from the three-body runs would have involved a hydrodynamic encounter between two of the stars, likely forming a merged object either bound or unbound to the third star. The remaining exchanges would have been clean, meaning we expect a binary to be produced without any hydrodynamic processes playing an important part. A very small fraction of encounters would have resulted in the ejection of the binary from a typical globular cluster core. In an equivalent diagram for a harder binary, the relative cross section for hydrodynamic 
events would be larger, with fewer clean encounters occurring.

We are thus left with the following picture. Binaries are fed into the core of a globular cluster and are either broken up immediately or harden progressively, receiving larger kicks at each succesive encounter, as the binary becomes more tightly bound. Eventually, the binary will be sufficiently tight that some form of merger is likely, unless the system contains two compact objects, in which case the system may well be ejected from the globular cluster. This evolutionary scenario is considered in more detail in McMillan et al. 1992, Davies (1995), and Davies \& Benz (1995).

\section{Encounters between Two Binaries}

Encounters between two binaries will be more important than encounters between binaries and single stars if the binary fraction is larger than $\sim 10 \%$, which it might be in the cores of some globular clusters. Relatively little work has been carried out on binary-binary encounters (see Bacon et al. 1995, and references contained therein). Such encounters may lead to a relatively large number of mergers, and triple systems.

\section{Summary}

Collisions happen in globular clusters and galactic nuclei. In globular clusters

- Encounters involving binaries may be important.

- Collisions may produce the stellar exotica that we see (LMXBS etc.).

- At least half of encounters between two single stars produce merged objects.

- Wide binaries $\Longrightarrow$ broken up.

- Hard binaries $\Longrightarrow$ harder (Heggie's Law)

- Massive stars exchange into binaries.

- Really hard binaries $\Longrightarrow$ stellar exotica

- The relative importance of binary/single and binary/binary encounters is a function of binary fraction. Binary/binary encounters will generally only be important if binary fraction $\geq 30 \%$.

- Better modelling of globular clusters and binary/binary cross sections are required and will come in the near future.

In galactic nuclei

- Many more simulations of stellar encounters are required.

- Very high velocity, head on encounters between main-sequence stars lead to their complete disruption. 
- Grazing encounters between two main-sequence stars lead to some mass loss but not complete disruption and leave the two stars unbound.

\section{Acknowledgements}

The support of a Royal Society URF is gratefully acknowledged. I thank the IAU for providing financial aid to attend IAU174.

\section{References}

Auriere M., Ortolani S., Lauzarel G., 1990, Nature, 344, 638

Bacon D., Sigurdsson S., Davies M. B., 1995, MNRAS, submitted

Bailyn C. D., Grindlay J. E., 1987, ApJ, 316, L25

Bailyn C. D., Grindlay J. E., Garcia M., 1990, ApJ, 357,L35

Bailyn C. D., et al. 1995, in preparation

Benz W., Bowers R. L., Cameron A. G. W., Press W. H., 1989, ApJ, 348, 647

Benz W., Hills J. G., 1987, ApJ, 323, 614

Benz W., Hills J. G., 1992, ApJ, 389, 546

Cannon R. C., 1993, MNRAS, 263, 817

Cleary P. W., Monaghan J. J., 1990, ApJ, 349, 150

Cohn, H., 1980, ApJ, 242, 765

Cool A., 1993, PhD thesis, Harvard University

Cool A., Grindlay J., Cohn H., Lugger P., Slavin S., 1995, ApJ, 439, 695

Davies M. B., 1995, MNRAS, 276, 887

Davies M. B., Benz W., 1995, MNRAS, 276, 887

Davies M. B., Benz W., Hills J. G., 1992, ApJ, 401, 246

Davies M. B., Benz W., Hills J. G., 1993, ApJ, 411, 285

Davies M. B., Benz W., Hills J. G., 1994, ApJ, 424, 870

de Young D. S., 1968, ApJ, 153, 633

Fabian, A., Pringle, J. E., Rees, M. J., 1975, 172, 17p

Grindlay J., Cool A., Callanan P., Cohn H., Lugger P., Bailyn C., 1995, ApJ, in press

Guhathakurta, P., Yanny B., Schneider D. P., Bahcall J. N., 1996, ApJ, submitted

Hartwick F. D. A., Hesser J. E., 1972, ApJ, 175, 77

Hernquist L., Goodman J., 1991, ApJ, 378, 637

Hut P., 1994, ApJS, 55, 301

Hut P., McMillan S., Romani R. W., 1992, ApJ, 389, 527

Kulkarni, S. R., Narayan, R., Romani, R. W., 1990, ApJ, 356, 174

Landau L., Lifschitz E., 1962, Quantum Mechanics (London: Wiley)

Lai D., Rasio F. A., Shapiro S. L., 1993, ApJ, 412, 593

Lombardi J. C., Rasio F. A., Shapiro S. L., 1995, ApJ, submitted

Lynden-Bell, D., Wood, R., 1968, MNRAS, 138, 495

Manchester, R. N., Lyne, A. G., Robinson, C., D'Amico, N., Lim, J., 1991, Nature, 352, 219

Mathis J. S.,, 1967, ApJ, 147, 1050

Nemec J. M., Harris H. C., 1987, ApJ, 316, 172

Paresce F., et al, 1991, Nature, 352, 297

Rasio F. A.,, Shapiro S., 1991, ApJ, 377, 559

Ruffert M., Müller E., 1990, A\&A, 238, 116

Seidl F. G. P., Cameron, A. G. W. 1972, AP Space Sci, 15, 44

Sigurdsson S., Phinney E. S. P., 1995, ApJS, 1995, 99, 609

Taam R. E., Bodenheimer P., 1989, ApJ, 337, 849

Yanny B., Schneider D. P., Bahcall J. N., Guhathakurta, P., 1994, ApJ, 435, L59 